\title{
Plasma virtual roughness elements for cross-flow instability control
}

\author{
Kwing-So Choi ${ }^{1}$. Jung-Hoon $\mathrm{Kim}^{1}$
}

Received: 2 May 2018 / Revised: 31 August 2018 / Accepted: 31 August 2018 / Published online: 1 October 2018

(c) The Author(s) 2018

\begin{abstract}
Recent experiments and numerical simulations demonstrated that discrete roughness elements can be used to control crossflow instability over a swept wing. Here, the application of this passive technique requires a row of thin cylindrical elements of a few microns high immediately downstream of the leading edge to excite the subcritical modes of cross-flow instability. By properly choosing the spanwise spacing of these roughness elements, one can suppress the growth of most unstable modes, thereby delay transition. However, this passive technique of controlling cross-flow instability is very sensitive to the size (diameter and height), shape and location of discrete roughness. To mimic the discrete roughness elements and to be able to adjust the roughness parameters dynamically, virtual roughness elements based on dielectric-barrier-discharge plasma actuators have been developed and tested. In this paper, we show the plasma-induced flow field of several different prototype virtual roughness elements for cross-flow instability control, by describing the mechanisms of vortex generation from the virtual roughness elements through an interaction with the incoming laminar boundary layer.
\end{abstract}

\section{Graphical abstract}
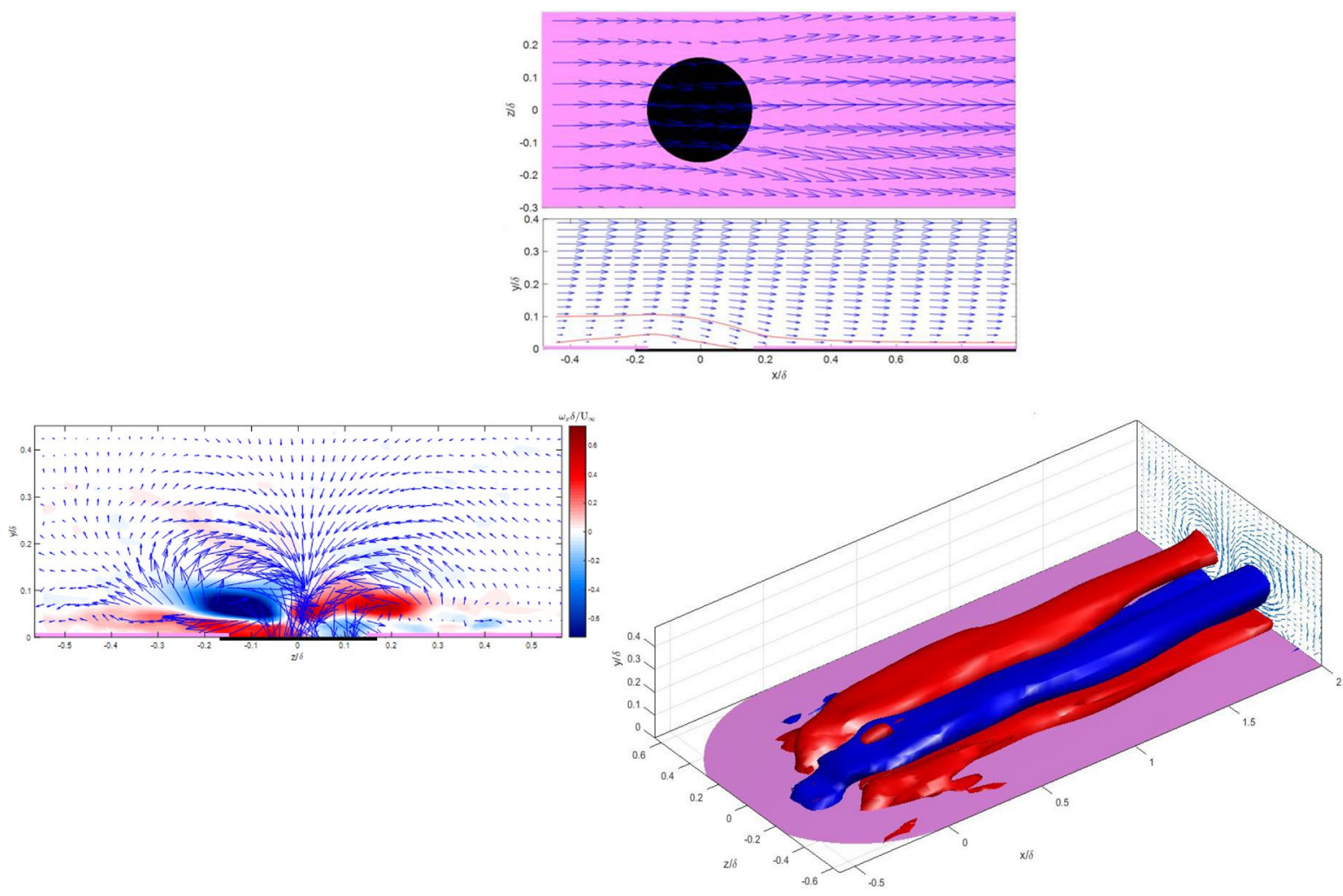

Extended author information available on the last page of the article 


\section{Introduction}

The boundary layer developing over a swept wing is highly three-dimensional. While the velocity component normal to the leading edge is reduced as the uniform flow approaches the swept wing, the velocity component parallel to the leading edge is not affected, therefore diverting the inviscid streamline outboard (Reed and Saric 1989). At the same time, the crosswise pressure gradient over the swept wing induces the cross-flow, the velocity perpendicular to the local inviscid flow vector, as the streamwise velocity within the boundary layer is reduced. Since the cross-flow velocity becomes zero at the wall as well as at the edge of the boundary layer, it has an inflection point, which is inviscidly unstable to flow disturbances. The transition to turbulence of the laminar boundary layer over a swept wing is usually dominated by the cross-flow instability rather than two-dimensional $\mathrm{T}-\mathrm{S}$ (Tollmien-Schlichting) wave instability (Saric et al. 2003). The cross-flow instability will manifest itself in co-rotating stationary vortices when the freestream turbulence level is low (less than $0.15 \%$ ), such as in-flight tests. In a flow where the turbulence level is high (greater than $0.15 \%$ ), on the other hand, travelling vortices will develop over a swept wing. In other words, stationary vortices are initiated by the surface roughness, while the travelling waves are triggered by the turbulence vorticity in the freestream (Bippes 1999).

Conducting wind tunnel experiments, Saric et al. (1998) have developed a passive technique of controlling the crossflow instability by placing discrete roughness elements in the area immediately downstream of the aerofoil's leading edge. An NFL (2)-0415 aerofoil was used in the tests, which was set at $45^{\circ}$ sweep angle and $\mathrm{a}-4^{\circ}$ angle of attack. This created a favourable pressure gradient to develop a crossflow over the suction side of the aerofoil, while suppressing the $\mathrm{T}-\mathrm{S}$ waves. This is important for this passive technique to work as it can only affect the cross-flow instability. The aerofoil surface was polished to a $0.25-\mu \mathrm{m}$ RMS finish, over which cylindrical-shaped roughness elements were placed at $2.3 \%$ chord from the leading edge. When the roughness elements were placed in $18 \mathrm{~mm}$ spacing, the most amplified stationary mode of cross-flow instability with the wavelength of $12 \mathrm{~mm}$ was suppressed. However, they have excited the first super-harmonic ( $9 \mathrm{~mm}$ wave length) at the same time, which was also very unstable. When they tested the thin cylindrical roughness elements in $8 \mathrm{~mm}$ spacing and $6 \mu \mathrm{m}$ high at the chord Reynolds number of $2.4 \times 10^{6}$, the transition point was delayed by $13 \%$ past the minimum pressure location of the aerofoil where the natural transition takes place. Here, a strong early growth of the $8-\mathrm{mm}$ mode effectively suppressed the growth of very unstable $12 \mathrm{~mm}$ and $9 \mathrm{~mm}$ modes, which resulted in a transition delay.
This rather counter-intuitive technique for cross-flow instability control involves forcing of subcritical cross-flow instability modes, thereby modifying the mean velocity profile to become less unstable (Wassermann and Kloker 2002). Unlike the most unstable mode of the cross-flow instability, the subcritical modes do not continue to grow in the downstream, although the initial growth could be greater. In choosing the spanwise spacing of discrete roughness elements, it is important to know that the subharmonics created by the roughness elements are not destabilised (Reibert et al. 1996). In other words, discrete roughness elements do not destabilise modes with greater wavelengths than themselves. Therefore, the spacing between the discrete roughness elements is usually set to $2 / 3$ of the wavelength of most amplified stationary cross-flow instability mode (Saric et al. 1998).

Many experimental studies have been carried out to repeat and confirm Saric's tests. However, the success rate in passive control of cross-flow instability using discrete roughness elements has been rather low. This is partly because this passive control technique is very sensitive to the size (diameter and height), shape and location of discrete roughness (Serpieri at al. 2017). Indeed, Kurz and Kloker (2014) showed that the most amplified stationary cross-flow mode scales super-linearly with the roughness height. In wind tunnel tests, for example, several thin films must be added to the discrete roughness elements to adjust the roughness height when the flow conditions are changed or uncertain, which is not always possible during flight. This is where the idea of using plasma virtual actuators to mimic the discrete roughness elements came from.

We have developed several different types of dielectric-barrier-discharge (DBD) plasma actuators for cross-flow control by mimicking the discrete roughness elements, which we call plasma virtual roughness elements. Unlike passive discrete roughness elements used by Saric and the co-workers, the equivalent roughness height of plasma virtual roughness elements can be actively controlled by adjusting the power input. The spanwise spacing between the plasma virtual roughness elements can also be adjusted in real time by switching to different rows of plasma actuators. As a part of initial characterisation, we have carried out an investigation of plasma-induced flow field of some of prototype virtual roughness elements in a two-dimensional laminar boundary layer. Here, we describe the mechanisms of vortex generation from the plasma virtual roughness elements, which could interact with stationary streamwise vortices for cross-flow instability control. 


\section{Background}

\subsection{Dielectric barrier discharge (DBD) plasma actuators}

Dielectric barrier discharge (DBD) plasma actuators are new breed of flow control actuators based on low-temperature plasma (Moreau 2007; Corke et al. 2010; Kriegseis et al. 2016). They are fast-acting, all-electric actuators without moving parts, consisting only of a pair of (upper and lower) electrodes sandwiching a dielectric sheet (Wang et al. 2013), see Fig. 1. When high AC voltage is applied, air surrounding the plasma actuator is ionized, resulting in a body force acting on the ambient air in the presence of the electric field produced by the actuator geometry (Corke et al. 2010). In the first half of the AC cycle (negative-going cycle), electrons are pushed away from the upper electrode towards the near-by dielectric surface to accumulate the negative charge. Then the electrons over the dielectric surface are attracted towards the upper electrode in the second (positive-going) AC half cycle (Corke et al. 2010). Here, the movement of positive ions is opposite to that of electrons, contributing to the momentum coupling with the surrounding air. The net body force produced by the plasma actuator over the full AC cycle is always in a direction away from the upper electrode, which is due to the additional involvement of negative ions in the momentum coupling by DBD plasma actuators (Boeuf et al. 2009). The plasma-induced jet flow can be tailored in any shape and direction, by configuring the upper and lower electrodes appropriately. DBD plasma actuators have been used as virtual devices, such as vortex generators, flaps, travelling wave makers and tip-clearance seals, where significant improvements in aerodynamic and flow control performance have been made (Choi et al. 2015).

\subsection{Comb-type plasma actuators}

Comb-type plasma actuators have been developed for crossflow instability control over a cone at Mach 3 (Schuele 2010; Schuele et al. 2013). The upper electrode (anode) has a shape of comb teeth, while the lower electrode (cathode bus) is made of a cross bar located beneath the dielectric

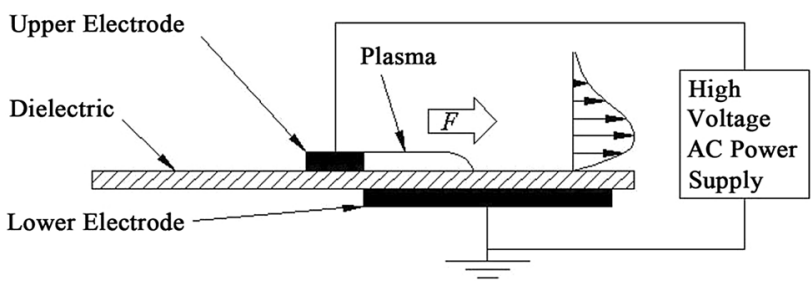

Fig. 1 Typical DBD plasma actuator configuration sheet. When the plasma actuators are turned on, plasma is formed on the outer edge of the comb teeth, creating counter-rotating vortices in the streamwise direction (Jukes and Choi 2013). This simulates the flow over discrete roughness elements, where counter-rotating pairs of vortices develop around the neck of the roughness elements. A $14^{\circ}$ rightcircular cone was machined out of ceramic (Macor) to a thickness of about $0.4 \mathrm{~mm}$, which acted as dielectric for DBD plasma actuators. Conductive silver epoxy was filled inside the cone, to which high $\mathrm{AC}$ voltage $\left(5 \mathrm{kV}_{\mathrm{p}-\mathrm{p}}\right.$ at $\left.5 \mathrm{kHz}\right)$ was applied. The external cone surface was copper deposited to a $0.05-\mu \mathrm{m}$ thickness, which was then etched into a comb shape by photo-lithography technique. Depending on the instability modes to be investigated, the spacing of the comb's teeth varied from $150 \mu \mathrm{m}$ (which corresponds to the wave number of subcritical mode of cross-flow instability, $m=68$ ) to $227 \mu \mathrm{m}$ (wave number of the critical mode, $m=45$ ). The experimental results seem to suggest that the stationary cross-flow modes are receptive to the comb-type plasma actuators. However, the Pitot tube measurements over the cone showed an increase in the total pressure for both critical $(m=45)$ and subcritical $(m=68)$ wave number cases, confirming that the transition point has been shifted forwards by the plasma actuators.

Similar comb-type plasma actuators were developed and for cross-flow instability control over a swept wing (Serpieri et al. 2017; Venkata 2016). The upper (exposed) electrode of the plasma actuators was made of $5 \mathrm{~mm}$ wide and $60 \mu \mathrm{m}$ thick copper tape, attached over a $60-\mu m$-thick Kapton dielectric sheet. The comb-shaped lower (concealed) electrode was made of silver-based conducting paint, sprayed over a stencil. The spanwise wave length $\lambda_{z}$ of the comb teeth was $7 \mathrm{~mm}$, corresponding to that of subcritical mode of crossflow instability. The plasma actuator was placed at the $2.5 \%$ chord from the leading edge of a slightly modified NACA 66-018 aerofoil at a swept angle of $45^{\circ}$ and an angle of attack of $3^{\circ}$. The applied AC voltage was $6 \mathrm{kV}$ at $3 \mathrm{kHz}$. Unfortunately, the transition front was moved upstream when the plasma actuators were operated, therefore promoted the transition by $10 \%$ chord. The total thickness of this plasma actuator was rather large $(125 \mu \mathrm{m})$ compared to the boundary layer thickness at $2.5 \%$ chord. It is claimed, however, that this should not affect the cross-flow instability as it is not quite receptive to a two-dimensional roughness. The reduction of the transition front width suggests the presence of the travelling modes, which may dominate the boundary layer and wash out the stationary modes, thus causing early transition (Serpieri et al. 2017; Venkata 2016).

\subsection{Ring-type plasma actuators}

Ring-type plasma actuators of 3-mm aperture diameter were investigated by Humble et al. (2013) for controlling 
cross-flow dominated boundary layer. The actuators consisted of upper electrodes made of etched gold-plated copper, which were attached to 0.5 -mm-thick polyimide dielectric sheets. The AC supply with $5 \mathrm{kV}$ peak-to peak at $5 \mathrm{kHz}$ was applied to the lower electrodes of the same aperture diameter, while the upper electrodes were connected to ground. A PIV measurement in a quiescent air indicates that the plasma actuator induced a wall-normal jet flow that is directed towards the centre of the actuator. The direction of flow field is opposite to that of Santhanakrishnan and Jacob (2007) on a 12.7-mm-diameter ring-type plasma actuator. They also tested a 7-mm diameter actuator, which showed that the induced flow was away from the actuator centre. These results suggest that the direction of induced wallnormal jet from ring-type actuators depends on the actuator diameter. The observed flow reversal for small ring-type actuators seems to be due to a cancellation of the electric field inside the actuators, therefore the plasma discharge may develop preferentially on the outer boundary of the upper electrode.

\subsection{Sandwich-type plasma actuators}

Sandwich-type plasma actuators were developed and tested by Moralev et al. (2018). These actuators have an upper and a lower electrode, sandwiching the comb-shape electrode to stabilise the plasma discharge from the upper electrode. The concept of sandwich-type plasma actuators is similar to that of comb-type plasma actuators used (Schuele et al. 2013; Venkata 2016), but the teeth of the comb, $2 \mathrm{~mm}$ wide with $4 \mathrm{~mm}$ spacing, in the sandwiched layer was connected to the ground together with the alumina ceramic layer at the bottom. Here, the upper electrode was made of a $20-\mu \mathrm{m}$-thick aluminium foil, where the AC voltage of $2-9 \mathrm{kV}$ at $7-200 \mathrm{kHz}$ was applied. PIV measurements demonstrate that the plasma discharge structure was similar to that of conventional plasma actuator (i.e. without a sandwiched comb layer), except that the discharge position was very stable. They also show a formation of steady streamwise vortices immediately downstream of the sandwich-type plasma actuators.

To investigate the effect of plasma discharge on the crossflow instability modes, tests were carried out using a flat plate (Ustinov et al. 2017). Here, the spanwise pressure gradient was induced by a wall bump placed over the ceiling of the wind tunnel. The swept angle of the flat plate and the wall bump was $35^{\circ}$, where the freestream velocity was $31.9 \mathrm{~m} / \mathrm{s}$. The corresponding Reynolds number was $2.1 \times 10^{6}$ per metre, where the freestream turbulence level was $0.02 \%$. The sandwich-type plasma actuators with the comb's teeth spacing, $\lambda=4 \mathrm{~mm}$ were placed at $90 \mathrm{~mm}$ from the leading edge of the flat plate. They were operated by $3.6 \mathrm{kV}$ AC voltage at $60 \mathrm{kHz}$. Modulation of tangential and crossflow velocities along the leading edge of the flat plate at a small distance from the wall surface were measured using $\mathrm{V}$-shaped, hot-wire anemometry. The results indicated that the sandwich-type plasma actuators with $4 \mathrm{~mm}$ teeth spacing have excited a steady instability mode with a spanwise wavelength of $5 \mathrm{~mm}$. Here, the linear instability analysis predicts that the spanwise wavelength of most unstable cross-flow instability is $7.5 \mathrm{~mm}$. However, the cross-flow instability was promoted by the discharge from the sandwich-type plasma actuators, shifting the transition point upstream.

\section{Experiments}

\subsection{Plasma virtual roughness elements}

Plasma actuators that have been designed are shown in Fig. 2. Having only one tooth in each actuator, the asymmetric-cross and T-type plasma actuators can be considered as the simplest form of the comb-type plasma actuators. The main objective for testing these designs is to find out the optimum configuration of the plasma actuators, particularly in the positioning of upper and lower electrodes to simulate a discrete roughness element for cross-flow instability control.

They were fabricated by printing the electrodes using silver paint over both sides of the Cirlex (thick Kapton) dielectric sheet of $0.25 \mathrm{~mm}$ thickness. The thickness of the upper (shown in red in Fig. 2) and lower (shown in black) printed electrodes was about $2 \mu \mathrm{m}$, thus the plasma electrodes will not trip the laminar boundary layer. It should be noted that the upper and lower electrodes are overlapped by $0.5 \mathrm{~mm}$ for the asymmetric-cross plasma actuators, see Fig. 2a, while
Fig. 2 Geometries of the a asymmetric cross, $\mathbf{b}$ T-type and c ring-type plasma actuators. The upper electrode is shown in red, while the lower electrode is in black. All measurements are in millimetres

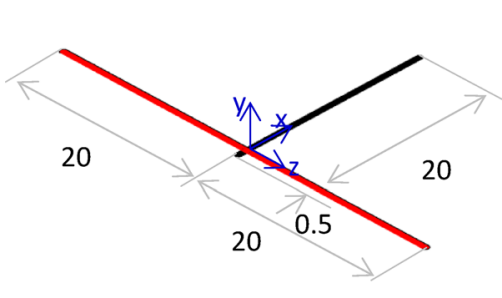

(a)

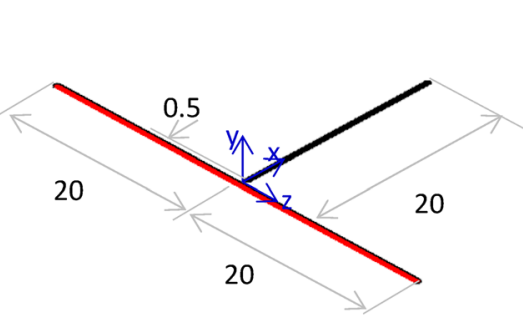

(b)

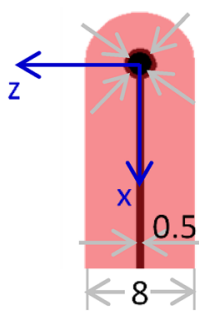

(c) 
the T-type plasma actuators have a small gap of $0.5 \mathrm{~mm}$ in between.

\subsection{Wind tunnel measurements}

Experiments were carried out in an open return wind tunnel with a test section of $1.5 \mathrm{~m} \times 0.3 \mathrm{~m} \times 0.3 \mathrm{~m}$. A 1-m-long and 10 -mm-thick flat plate with a super-elliptic leading edge was placed $0.1 \mathrm{~m}$ above the lower wall of the test section. Plasma actuators were attached to a flat plate at $0.49 \mathrm{~m}$ from the leading edge, where a two-dimensional laminar boundary layer was developed. The measured velocity profile is shown in Fig. 3, which is compared with the Blasius profile. With the shape factor of $H=2.5$, there is a slight deviation from the Blasius profile, which may well be due to the virtual original of the boundary layer development. The freestream velocity in the wind tunnel was $U_{\infty}=5.1 \mathrm{~m} / \mathrm{s}$, where the boundary layer thickness was $\delta=6.3 \mathrm{~mm}$ at the plasma actuator location. The freestream turbulence level was $0.25 \%$ at this speed. The Reynolds number was $1.7 \times 10^{5}$ based on the distance from the leading edge of the test plate. All plasma actuators were powered by an AC supply of sinusoidal wave form at $3.2 \mathrm{kV}$ (zero to peak) at $40 \mathrm{kHz}$.

The velocity field around the plasma actuators was measured using a two-dimensional PIV system, consisting of a Litron LDY302-PIV Nd:YLF laser with $15 \mathrm{~mJ}$ per pulse, a Vision Research Phantom V12 high-speed camera with a QUESTAR QM1 Short Mount Long-Distance Microscope, a National Instrument 80N77 Timer Box and a dedicated PC. Olive oil seeding particles of nominally $1 \mu \mathrm{m}$ diameter were introduced upstream of the wind tunnel contraction section from a TSI 9307-6 oil droplet generator via a seeding rake.

PIV measurements were made on several streamwise $(x-y)$ as well as spanwise $(y-z)$ planes, where the thickness of the laser light sheet was $1 \mathrm{~mm}$ and $2 \mathrm{~mm}$ in $x-y$ and $y-z$ planes, respectively. Successive image pairs were

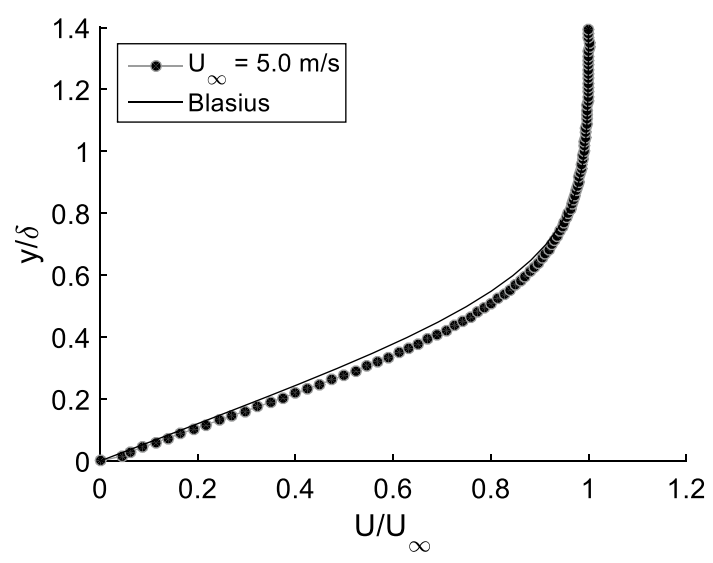

Fig. 3 Laminar boundary layer profile over a flat plate at the actuator position taken within a short interval of $75 \mu$ s at a repetition rate of $200 \mathrm{~Hz}$. The sampling period was $1.5 \mathrm{~s}$ for $x-y$ plane measurements and $2.0 \mathrm{~s}$ for $y-z$ plane. Here, the field of view was approximately $12 \mathrm{~mm} \times 3 \mathrm{~mm}(1050 \times 260$ pixels $)$. For spanwise velocity measurements, a $50 \mathrm{~mm} \times 50 \mathrm{~mm}$ high precision mirror was placed $200 \mathrm{~mm}$ downstream at $45^{\circ}$ to the freestream velocity so that the images could be taken from outside the wind tunnel. Typical streamwise distance between two successive spanwise planes was $2 \mathrm{~mm}$ for the asymmetric-cross and T-type plasma actuators and $1 \mathrm{~mm}$ for the ring-type plasma actuator, although some additional measurements were carried out in other planes near the electrodes. The total number of measurements was 12 and 16 , respectively, although not all velocity vectors are shown in Figs. 5, 9 and 12.

PIV image and data processing were carried out using Dantec DynamicStudio 2015a version. Here, each image was subtracted by the time-averaged image before the adaptive PIV cross-correlation algorithm was applied to compute the velocity vectors. The minimum and maximum interrogation area were set at 32 and 64 pixels in $x$ and $z$ directions, and 16 and 32 pixels in $y$ direction, both with $50 \%$ overlap. The size of grid, to which the time-average velocities were interpolated, was 16 pixels in $x$ and $z$ directions and 8 pixels in $\mathrm{y}$ direction, corresponding to $0.170 \mathrm{~mm}$ and $0.085 \mathrm{~mm}$ in $x-y$ planes and 0.182 and $0.091 \mathrm{~mm}$ in $y-z$ planes, respectively. The total PIV measurement error is expected to be equal to or less than 5\% of the local velocity (Westerweel 1997). The errors in the spanwise velocity measurements due to apparent translation of seeding particles from the centre towards the edge of the image should be less than $0.4 \%$ of the freestream velocity, or $0.02 \mathrm{~m} / \mathrm{s}$. Further details of the experiments and their error analysis can be found in Jukes and Choi (2013).

\section{Results and discussion}

\subsection{Asymmetric-cross plasma actuator}

Figure 4 shows the velocity vectors around the asymmetric-cross plasma actuator in the centre plane (i.e. along the lower electrode), where the red and black lines at $y / \delta=0$ indicate the location of the upper and lower electrodes, respectively. The velocity profile of the on-coming boundary layer remains unchanged until $x / \delta<-0.5$ (not shown here). As the boundary layer gets closer to the plasma actuator, however, the velocities are reduced by the plasma-induced flow, creating a reversal region near the wall, as shown in this figure.

A three-dimensional flow field around the asymmetriccross plasma actuator in the laminar boundary layer is given in Fig. 5, showing the spanwise velocity vectors and 
Fig. 4 Velocity vectors in the wall-parallel plane at $y=0.4$ $\mathrm{mm}(y / \delta=0.065)(\mathbf{a})$ and in the centre plane $(z / \delta=0)(\mathbf{b})$ with the asymmetric-cross plasma actuator. The upper electrode is shown in pink, while the lower electrode is in thick black. A streamline (shown in red) is shown to indicate the upstream effect of the actuator
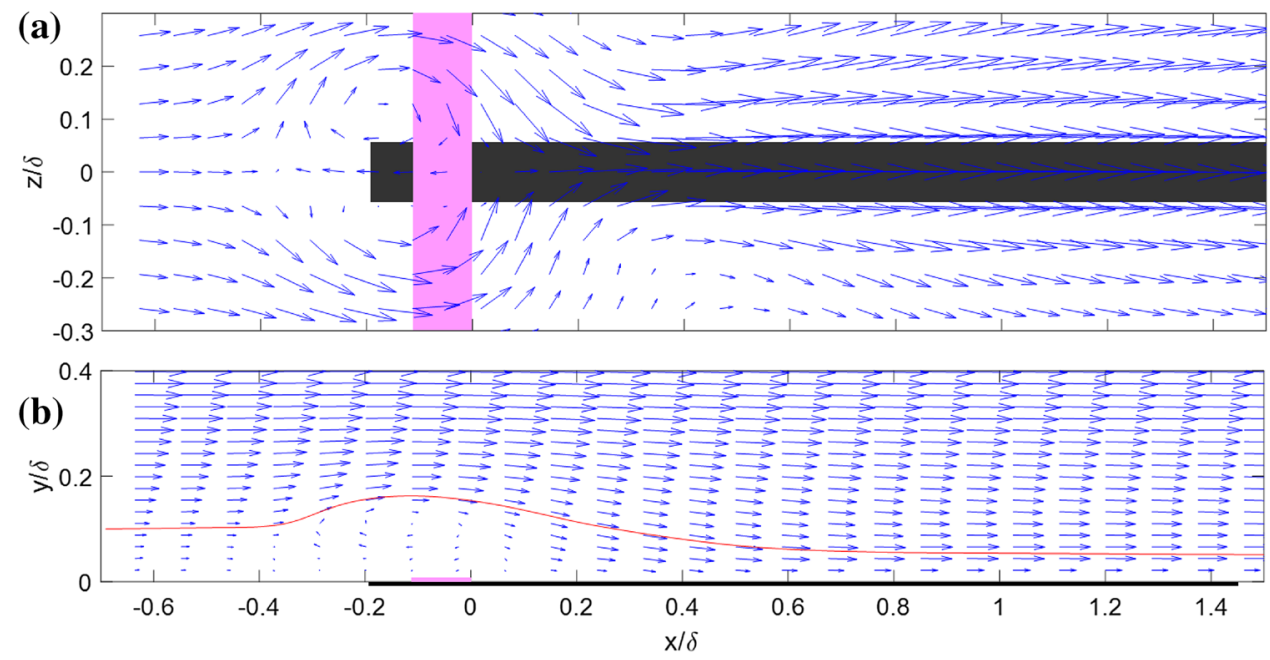

streamwise vorticity contours in eight spanwise planes. Also shown in the figure are velocity vectors in wall-parallel plane at $y / \delta=0.083$ (in grey colour) and those in the centre plane at $z / \delta=0$ (in green). Figure 5 shows that the flow diverges upstream of the plasma actuator at around $x / \delta=-0.5$, and converges immediately downstream at $x / \delta=0.2$, which has a strong similarity to a laminar boundary layer flow over a hemisphere, as shown by Acarlar and Smith (1987). It is therefore expected that a diverging-converging flow would result in a formation of hairpin vortices over the upstream recirculation region.

Figure 6 is a velocity vector plot in the spanwise plane at $x / \delta=-0.08$ taken from Fig. 5, showing that the spanwise flow accelerates as it converges towards the origin where the upper electrode crosses with the lower electrode, giving rise to an opposite-signed pair of vorticity regions at the height of $y / \delta=0.1$. A similar opposite-signed pair of vorticity regions, marked by $\mathrm{A}$ and $\mathrm{A}^{\prime}$ in Fig. 7, is also observed in a quiescent flow (not shown here). The opposite-signed vorticity layers are also observed in the near-wall region (see Fig. 6), indicating an initial development of necklace vortices. The counter-rotating necklace vortices are created upstream of the virtual roughness element, extending downstream far beyond $x / \delta=1.4$. The necklace vortices are also clearly shown in Fig. 6, marked by B and B' in Fig. 7. The thin region below $\mathrm{B}$ and $\mathrm{B}^{\prime}$ in Fig. 7 is secondary vorticity layers induced by the necklace vortices.

\subsection{T-type plasma actuator}

The upper electrode of the T-type plasma actuator does not overlap with the lower electrode; it stops short of the edge of the lower electrode. Therefore, the upstream effect of the T-type plasma actuator is expected to be much weaker than that of asymmetric cross-type plasma actuator whose upper electrode goes beyond the edge of the lower electrode.
Figure 8 shows the velocity vectors around the T-type plasma actuator in the centre plane (along the lower electrode), confirming that the recirculating flow region is barely visible even close to the plasma actuator.

A three-dimensional flow field around the T-type plasma actuator in the laminar boundary layer is given in Fig. 9, showing the spanwise velocity vectors and streamwise vorticity contours in seven spanwise planes. The streamwise vorticity associated with this flow field is shown in Fig. 10, where less significant divergence/convergence around the T-type plasma actuator results in smaller pair of vorticities (marked A and A' in Fig. 10). The necklace vortices are also slightly weakened, but is still seen extended downstream far beyond $x / \delta=1.4$ (marked B and B' in Fig. 10).

\subsection{Ring-type plasma actuator}

Figure 11 shows a velocity vector plot of the flow over a 2-mm-diameter ring-type plasma actuator in the centre plane. Streamlines drawn in the figure indicate that there is an upwash at the frontal edge of the ring, which is followed by a downwash towards the centre of the ring.

A three-dimensional flow field around the ring-type plasma actuator is given in Fig. 12, showing the spanwise velocity vectors and streamwise vorticity contours in eight spanwise planes. Also shown in Fig. 12 are velocity vectors in the wall-parallel plane at $y / \delta=0.083$ (in grey colour) and those in the centre plane, $z / \delta=0$ (in green). The streamwise vorticity associated with this flow field is shown in Fig. 14.

The ring-type plasma actuators with small diameter ( $d \lesssim 5 \mathrm{~mm}$ ) induce wall-normal flow towards the centre of the actuator (Humble et al. 2013), therefore the flow around this ring-type actuator is accelerated towards the lower electrode and spread out in the radial directions. This is displayed in Fig. 13 in the spanwise plane at $x / \delta$ $=-0.07$ (taken from Fig. 12), which shows the necklace 
Fig. 5 Spanwise velocity vectors with streamwise vorticity contours in a laminar boundary layer with the asymmetric-cross plasma actuator at $x / \delta=0$. The grey velocity vectors are shown in the wall-parallel plane at $y / \delta=0.083$, while green velocity vectors are in the centre plane, $z / \delta=0$. The upper electrode is shown in pink, while the lower electrode is in thick black.

Streamwise velocity vectors are reduce by a factor of 5 for clarity

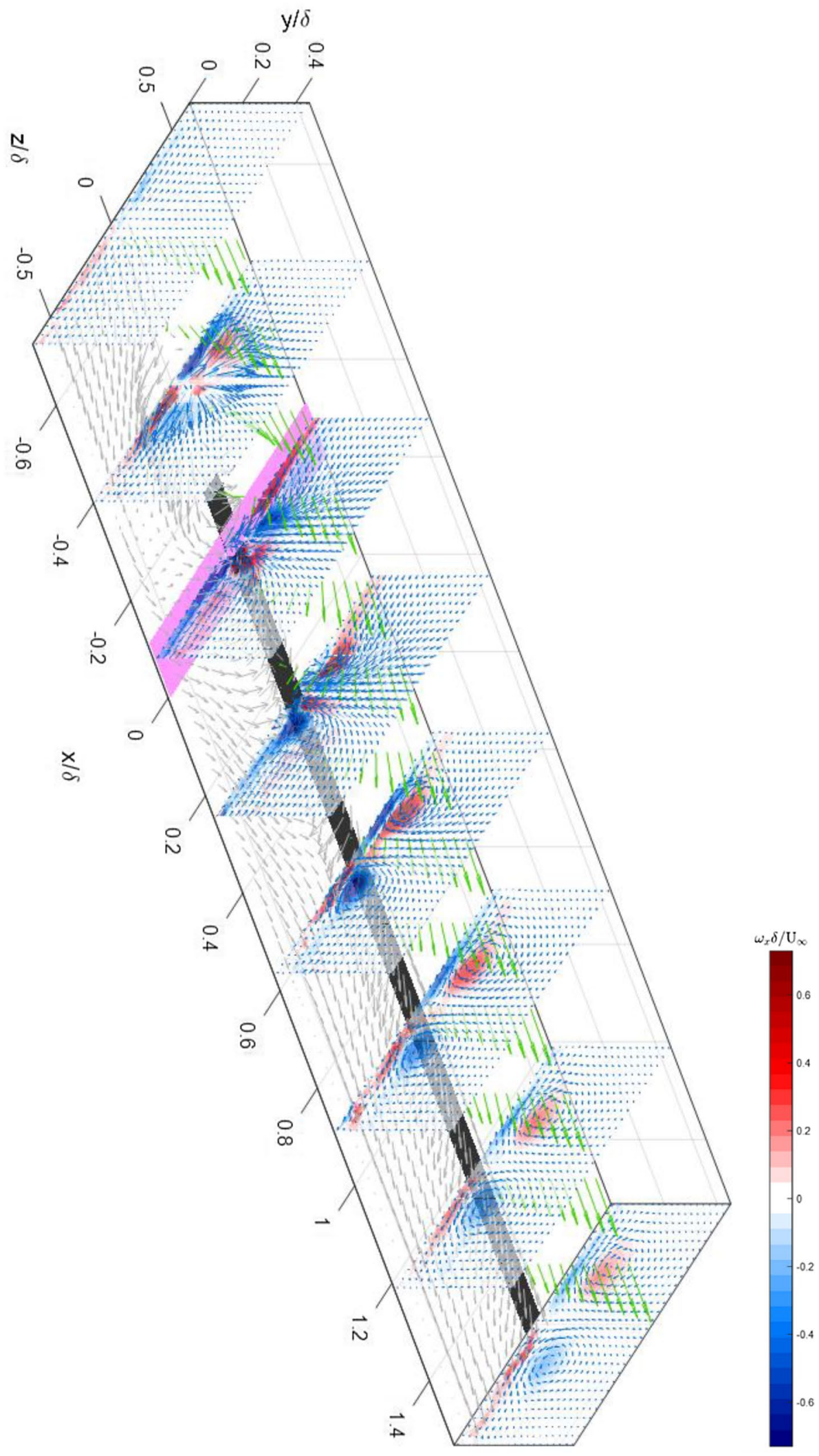


Fig. 6 Velocity vectors with vorticity contours with the symmetric-cross plasma actuator at $x / \delta=-0.08$. The flow is viewed from downstream
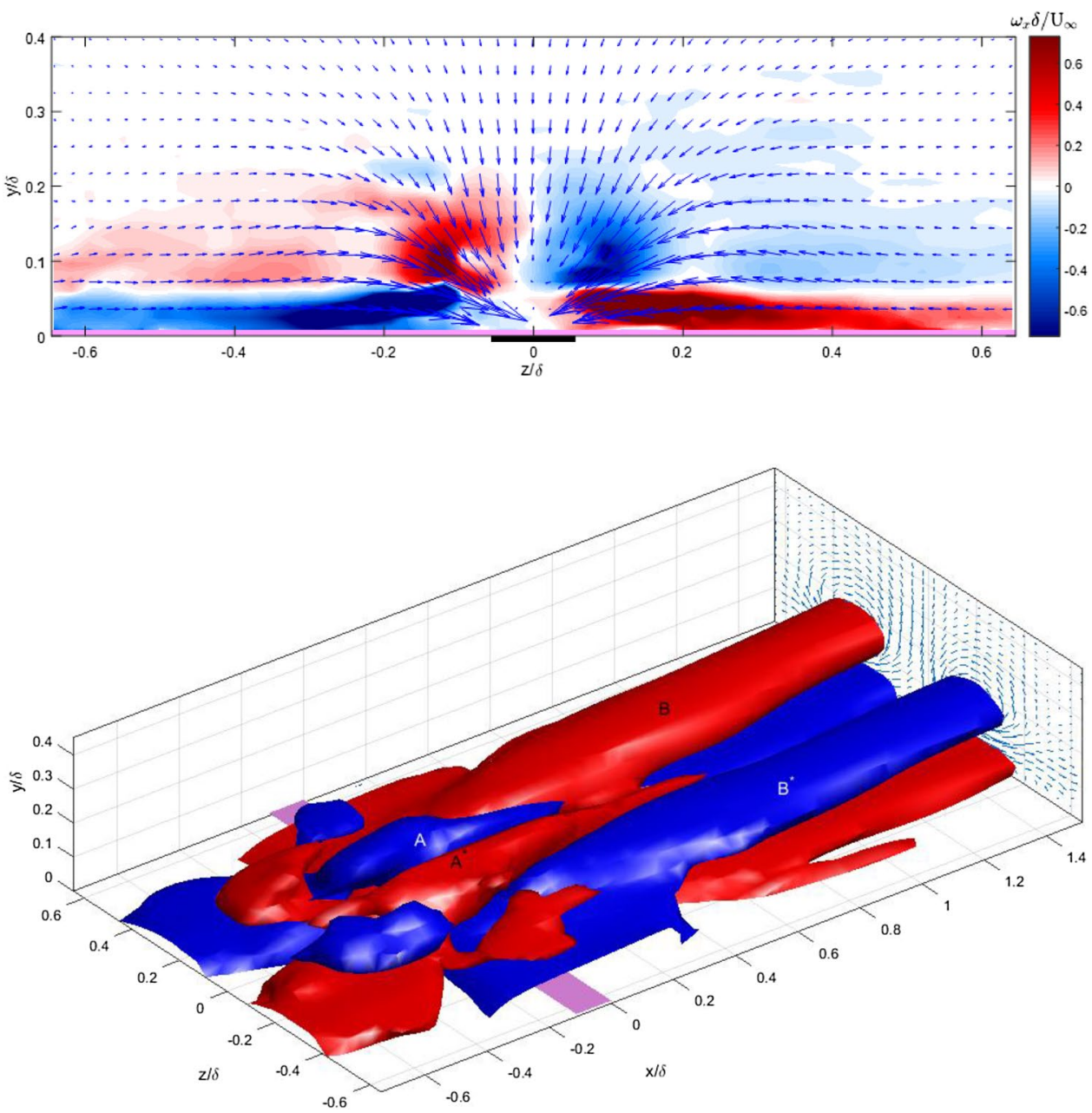

Fig. 7 Isosurface of the streamwise vorticity $\omega_{x} \delta / U_{\infty}=-0.18$ (blue) and 0.18 (red) with the asymmetric-cross plasma actuator. The upper electrode is shown in pink

Fig. 8 Velocity vectors in the wall-parallel plane at $y=0.4 \mathrm{~mm}(y / \delta=0.065)(\mathbf{a})$ and in the centre plane $(z / \delta=0)$ (b) with the asymmetric-cross plasma actuator. The upper electrode is shown in pink, while the lower electrode is in thick black. A streamline (shown in red) is shown to indicate the upstream effect of the actuator
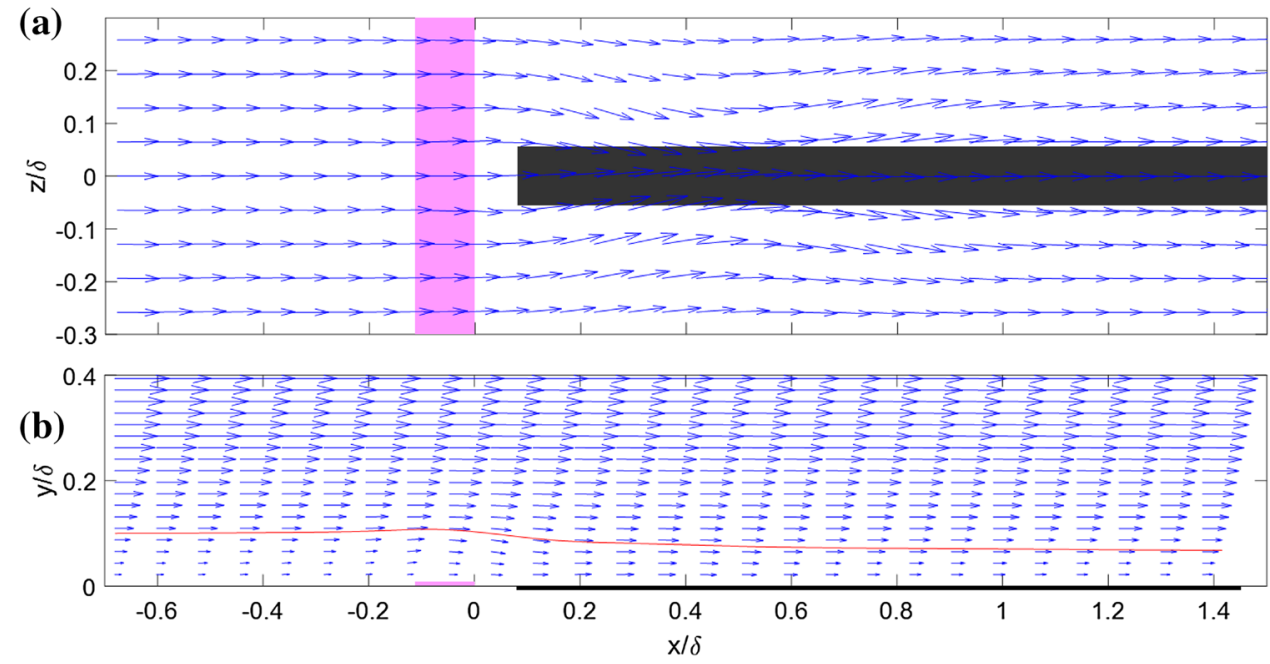

vortices and the secondary vortices over the ring-type plasma actuator without additional vorticity pair on top. This is also confirmed in Fig. 14, where there is no vorticity pair similar to those in Figs. 7 and 10 (marked by $A$ and $A^{\prime}$ ).
Since the virtual roughness element created by a ringtype plasma actuator has a lower profile than that by the asymmetric-cross or T-type plasma actuators, it is expected that the hairpin vortices are either very weak or not present at all. For example, there are no hairpin vortices in a flow 
Fig. 9 Spanwise velocity vectors with streamwise vorticity contours in a laminar boundary layer with the T-type plasma actuator at $x / \delta=0$. The grey velocity vectors are shown in the wall-parallel plane at $y / \delta$ $=0.083$, while green velocity vectors are in the centre plane, $z / \delta=0$. The upper electrode is shown in pink, while the lower electrode is in thick black. Streamwise velocity vectors are reduced by a factor of 5 for clarity

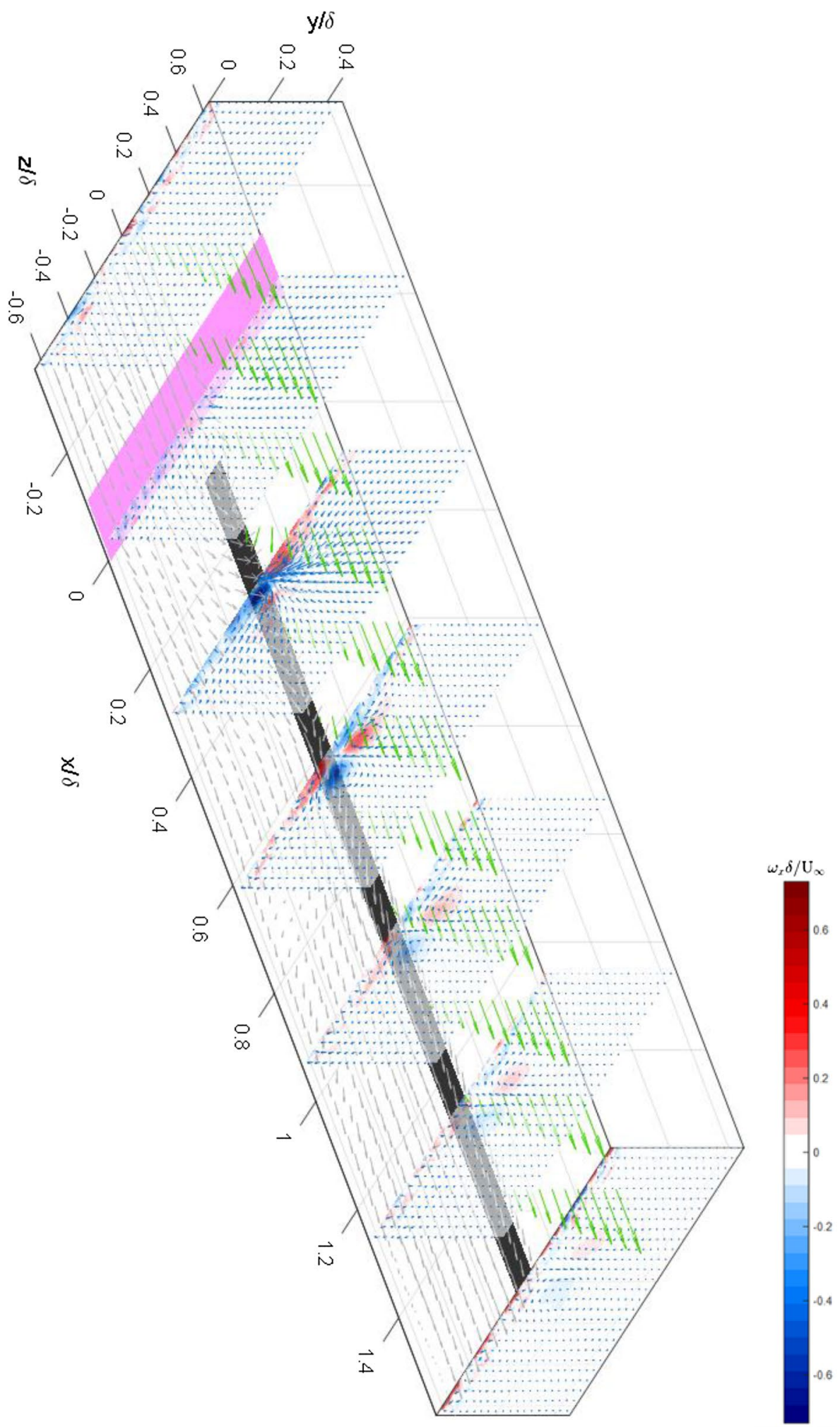

over a smooth circular disk, as shown in a DNS study by Kurz and Kloker (2016). The torus-shaped flow structure associated with the induced flow towards the centre of the ring-type plasma actuator (see Fig. 13) is very similar to that in Fig. 10 of Humber et al. (2013). This flow structure helps establish a pair of necklace vortices around the actuator without shedding hairpins, which are essential for crossflow instability control over a swept wing. 
Fig. 10 Isosurface of the streamwise vorticity $\omega_{x} \delta / U_{\infty}$ $=-0.11$ (blue) and 0.11 (red) with the T-type plasma actuator. The upper electrode is shown in pink

Fig. 11 Velocity vectors in the wall-parallel plane at $y=0.3 \mathrm{~mm}(y / \delta=0.048)(\mathbf{a})$ and in the centre plane $(z / \delta=0)(\mathbf{b})$ over a 2-mm ring-type actuator. The upper electrode is shown in pink, while the lower electrode is in thick black. Streamlines are shown in red to indicate the upstream effect of the actuator
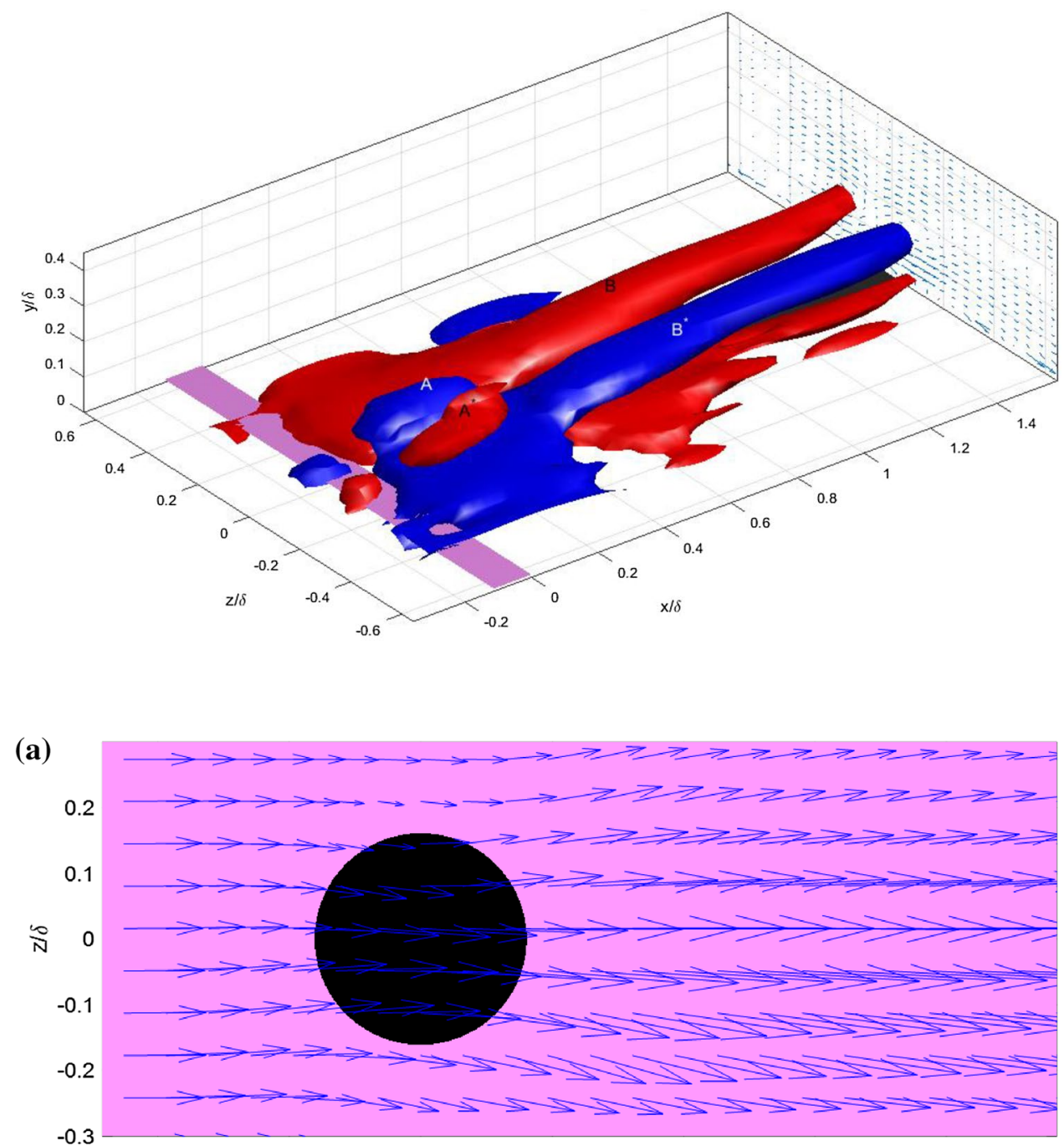

(b)

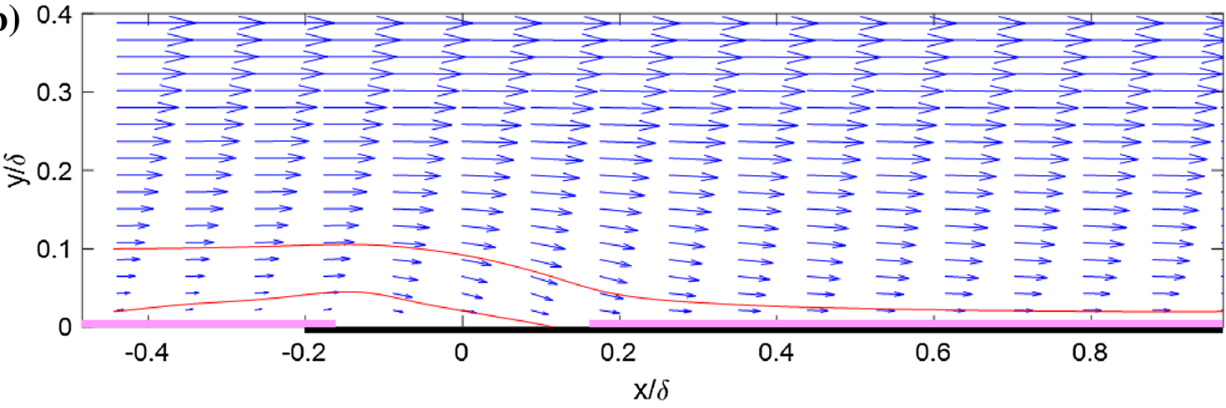

\section{Plasma virtual roughness elements for swept wing}

Based on the results presented in the previous section, we have designed and fabricated an array of ring-type plasma actuators on a 0.15 -mm-thick Cirlex sheet, over which upper and lower electrodes were printed with silver paint, see Fig. 15. With this array of plasma actuators, we were able to test different virtual roughness elements spacing of 3.5, 4, 4.5 or $6 \mathrm{~mm}$ at different chord-wide locations without reattaching the actuators over a swept wing. On installation to the airfoil, the ring-type actuators were located at $1.9 \%, 3.3 \%, 4.7 \%$ and $6.1 \%$ chord length from the leading edge. One can simply connect the power supply to each row of ring-type actuator to excite different cross-flow instability modes. The lower electrodes were designed such that only one row of plasma virtual roughness elements is connected at a time. AC sinusoidal voltages of up to $2.5 \mathrm{kV}$ zero-to-peak at $20 \mathrm{kHz}$ were applied 
Fig. 12 Spanwise velocity vectors with streamwise vorticity contours in a laminar boundary layer with the ring-type actuator at $x / \delta=0$. The grey velocity vectors are shown in the wallparallel plane at $y / \delta=0.083$, while green velocity vectors are in the centre plane, $z / \delta=0$. The upper electrode is shown in pink, while the lower electrode is in black. Streamwise velocity vectors are reduce by a factor of 5 for clarity

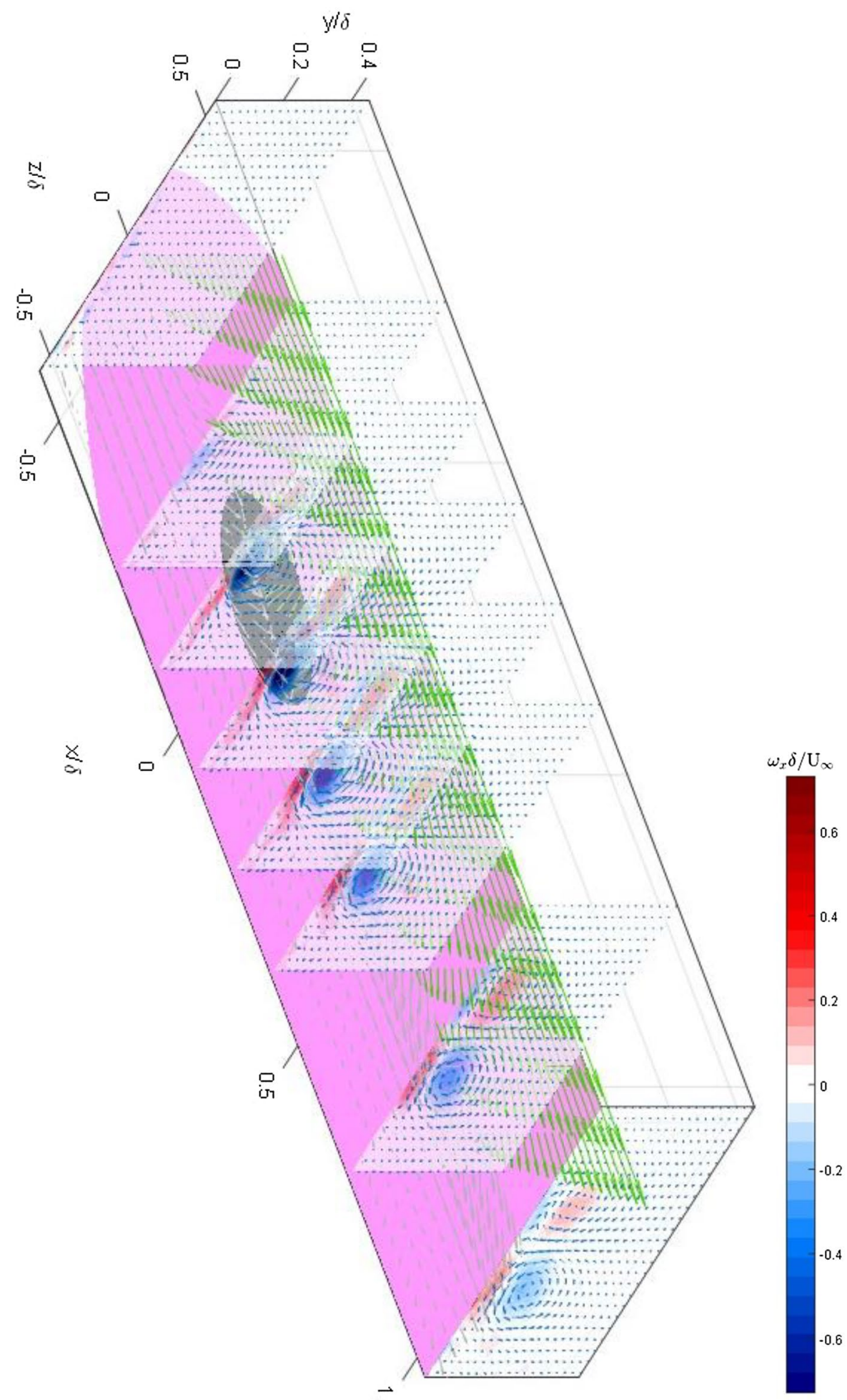

to the plasma actuator sheets by TREK power amplifier (model 30/20).

Preliminary tests were carried out to investigate into a possible transition delay using the plasma virtual roughness elements by controlling cross-flow instability over a swept wing. These tests took place at ONERA Toulouse, where an ONERA-D airfoil with $0.35 \mathrm{~m}$ chord length was set to $60^{\circ}$ of sweep angle and at the angle of attack of $-8^{\circ}$. The freestream velocity was kept at $70 \mathrm{~m} / \mathrm{s}$, corresponding to the Reynolds number of $1.6 \times 10^{6}$. The freestream turbulence level at this speed was $0.07 \%$. The actuator 
Fig. 13 Vorticity contours with spanwise velocity vectors at $x / \delta$ $=-0.07$. The upper electrode is shown in pink, while the lower electrode is in thick black. The flow is viewed from downstream
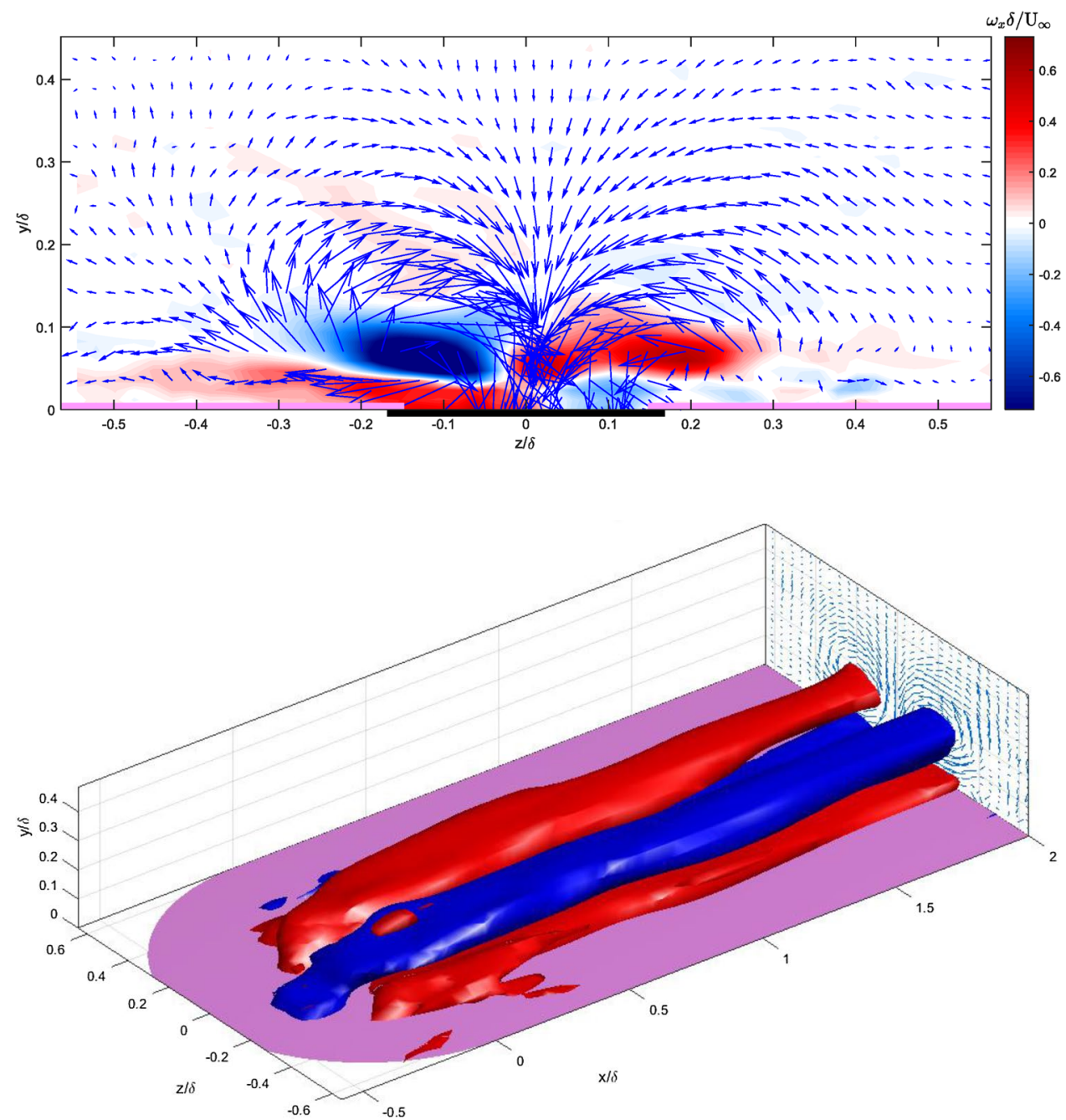

Fig. 14 Isosurface of the streamwise vorticity $\omega_{x} \delta / U_{\infty}$ $=-0.11$ (blue) and 0.11 (red) with the ring-type plasma actuator, $d=2 \mathrm{~mm}$ sheet was wrapped around the leading edge, as shown in Fig. 15c, to avoid any surface discontinuity.

Figure 16 shows the development of the RMS hot-wire voltages along the streamwise direction with and without plasma actuation. Here, these RMS values were obtained by integrating the fluctuating signals between $200 \mathrm{~Hz}$ and $10 \mathrm{kHz}$ in the voltage power spectra, removing the possible effect of probe/support vibrations as well as wind tunnel unsteadiness. The supplied voltage to the actuators varied between 1.1 and $1.5 \mathrm{kV}$ (peak to zero), where the input frequency was fixed at $15 \mathrm{kHz}$. It is clearly seen that the transition points, represented by the RMS peaks, were shifted upstream by plasma forcing for plasma excitation voltage over $1.3 \mathrm{kV}$. Shahriari et al. (2018) have carried out a DNS study of the cross-flow instability control using the same ring-type plasma actuators, and confirmed the experimental results given in Fig. 16. They indicated that plasma actuators with greater induced velocity would excite the subcritical modes more efficiently, so that they could delay the crossflow transition by attenuating both primary and secondary unsteady disturbances. Figure 17 shows the energy spectra at several wall-normal locations in the boundary layer at $x / c=10$, indicating an early growth of broadband energy between 2 and $7 \mathrm{kHz}$ with plasma forcing. The growth at these frequencies has been predicted by the linear instability analysis, see Kim et al. (2017). There are no signs of unsteadiness due to plasma actuators in these spectra at least below $10 \mathrm{kHz}$. Contrary to the hot-wire results in Serpieri and Kotsonis (2018), no significant electro-magnetic interference was observed either.

\section{Concluding remarks}

DBD plasma actuators are fast-acting, all-electric actuators without moving parts, consisting only of a pair of (upper and lower) electrodes sandwiching a dielectric sheet. These actuators can be integrated into aerodynamic bodies in design, or they can be retrofitted. However, the crucial weakness of the plasma actuators is their authority, as the maximum induced 
Fig. 15 Drawings of ring-type plasma actuators on a the upper and $\mathbf{b}$ the lower (ground) surfaces, which were attached to a swept aerofoil as shown in c. All measurements are in millimetres

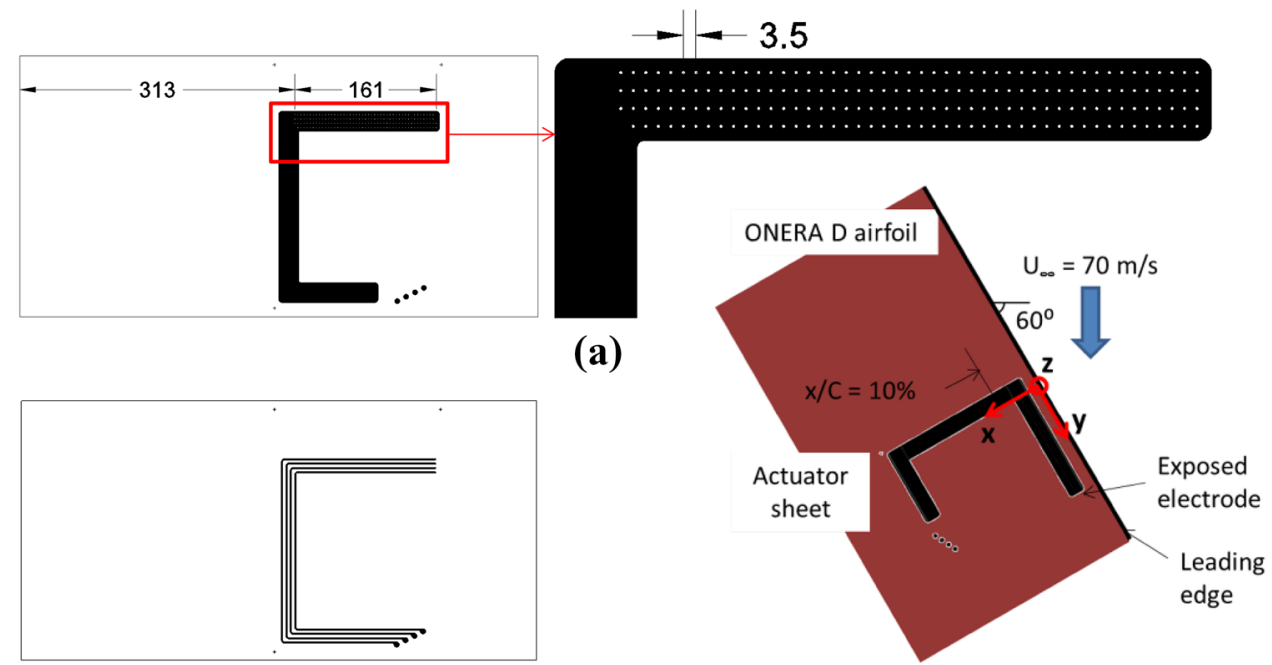

(b)

(c) velocity is limited to about $10 \mathrm{~m} / \mathrm{s}$. It is in this context that future applications of plasma actuators must be carefully considered. Flow instability control, for example, is an ideal application of DBD plasma actuators, because it does not

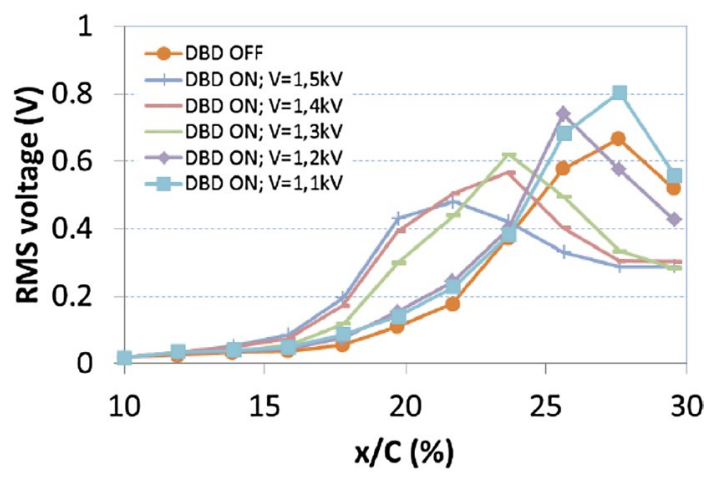

Fig. 16 Downstream development of RMS hot-wire signal with and without plasma forcing, from Kim et al. (2017)

demand a high authority on the flow.

For cross-flow instability control, it is important that the plasma-induced flow fields should be steady, so that only the targeted instability modes can be forced by the actuator without adversely affecting other modes. The plasma virtual roughness elements must also be thin enough not to trigger unstable disturbances when they are attached to the flow surface. Currently, three types of plasma actuator are available for cross-flow instability control-comb-type, ring-type and sandwich-type. All are simple in construction, but the induced flow fields are quite different to each other.

Here, we have investigated the induced flow field of asymmetric-cross and T-type plasma actuators in a laminar boundary layer with a view to determine the optimum configuration of the comb-type plasma actuators, particularly in the positioning of upper and lower electrodes to simulate a discrete roughness element for cross-flow instability control. Having only one tooth, asymmetric-

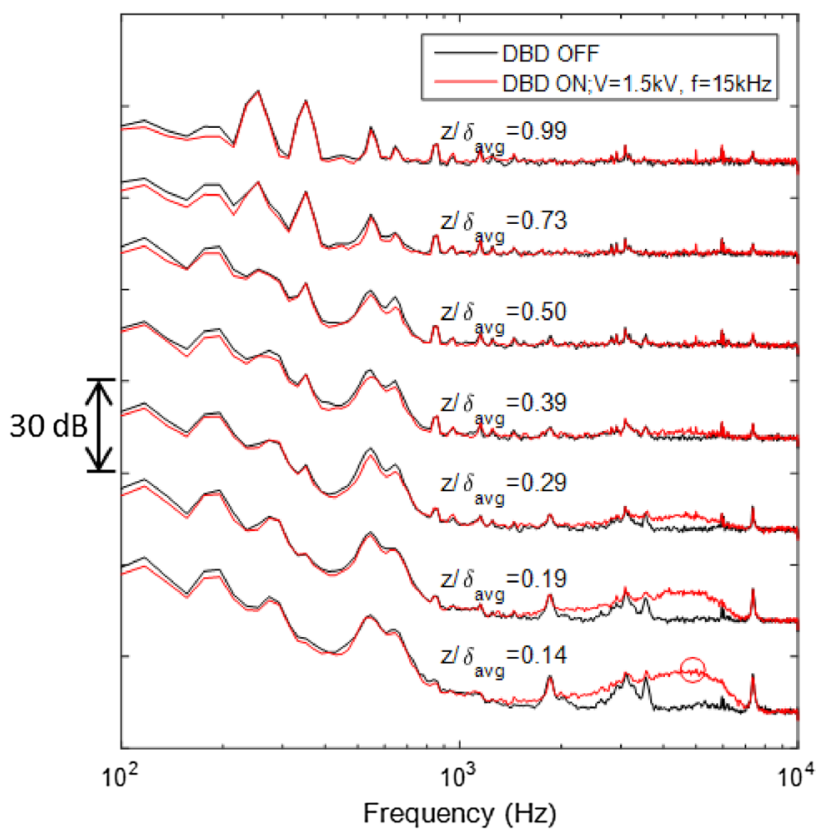

Fig. 17 Power spectra of velocity fluctuations in the boundary layer at $x / c=10 \%$, from Kim et al. (2017)

cross and T-type plasma actuators can be considered as the simplest form of the comb-type plasma actuators. These plasma actuators were then compared with ringtype plasma actuators. 
The flow field around an asymmetric-cross plasma actuator showed a flow divergence upstream of the plasma actuator, with a convergence immediately downstream, which has a strong similarity to a laminar boundary layer flow over a hemisphere shown by Acarlar and Smith (1987). It is therefore expected that a diverging-converging flow would result in a formation of hairpin vortices over the upstream recirculation region.

The upper electrode of the T-type plasma actuator does not overlap with the lower electrode; it stops short of the edge of the lower electrode. Therefore, the upstream effect of the T-type plasma actuator is expected to be much weaker than that of asymmetric cross-type plasma actuator whose upper electrode goes beyond the edge of the lower electrode. As a result, less significant divergence/ convergence flow was observed around the T-type plasma actuator.

The flow around a ring-type actuator was seen accelerated towards the lower electrode and spread out in the radial directions, confirming the results by Humble et al. (2013). It showed necklace vortices as well as secondary vortices over the ring-type plasma actuator, but without additional vorticity region on top. Since the flow induced by a ringtype plasma actuator has a lower profile than that by the asymmetric-cross or T-type plasma actuators, it is expected that the hairpin vortices are either very weak or not present at all, similar to the flow over a smooth circular disk (Kurz and Kloker 2016).

Preliminary results suggest that the rows of plasma virtual roughness elements could indeed influence the subcritical modes of cross-flow instability over a swept wing, confirming the results by Serpieri et al. (2017) who revealed distinct steady and unsteady forcing contributions of the plasma actuator on the boundary layer. To use these plasma virtual roughness elements for delaying the transition to turbulence, however, it is important that we should better understand the behaviour of plasma-induced flow and its effect on the three-dimensional boundary layer.

Dorr and Kloker (2017) recently proposed a unique approach to cross-flow instability control by placing a row of plasma actuator strips along the local mean-flow direction of a three-dimensional boundary layer. These plasma actuators apply body force against the cross flow to reduce the velocity component, while forcing the subcritical cross-flow instability mode. They claim that this forcing technique is more efficient than passive technique (e.g. discrete roughness elements) in exciting the required instability mode, thus is very suitable for active control using plasma actuators.

Acknowledgements This study was supported by the Engineering and Physical Sciences Research Council in the UK (grant number EP/ N018486/1) and EU FP7-AAT-2013.8-1-RTD-RUSSIA programme BUTEFLI "Buffet and transition delay control investigated within European-Russian cooperation for improved flight performance".
Open Access This article is distributed under the terms of the Creative Commons Attribution 4.0 International License (http://creativeco mmons.org/licenses/by/4.0/), which permits unrestricted use, distribution, and reproduction in any medium, provided you give appropriate credit to the original author(s) and the source, provide a link to the Creative Commons license, and indicate if changes were made.

\section{References}

Acarlar MS, Smith CR (1987) A study of hairpin vortices in a laminar boundary layer. Part 1 . Hairpin vortices generated by a hemisphere protuberance. J Fluid Mech 175:1-41

Bippes H (1999) Basic experiments on transition in three-dimensional boundary layers dominated by crossflow instability. Prog Aerosp Sci 35(4):363-412

Boeuf JP, Lagmich Y, Pitchford LC (2009) Contribution of positive and negative ions to the electrohydrodynamic force in a dielectric barrier discharge plasma actuator operating in air. J Appl Phys 106:023115

Choi K-S, Jukes TN, Whalley RD, Feng L, Wang J, Matsunuma T, Segawa T (2015) Plasma virtual actuators for flow control. J Flow Control Meas Visual 3:22-34

Corke TC, Enloe CL, Wilkinson SP (2010) Dielectric barrier discharge plasma actuators for flow control. Annu Rev Fluid Mech 42:505-529

Dorr PC, Kloker MJ (2017) Crossflow transition control by upstream flow deformation using plasma actuators. J Appl Phys 121:063303

Humble RA, Craig SA, Vadyak J, McClure PD, Hofferth JW, Saric WS (2013) Spatiotemporal structure of a millimetric annular dielectric barrier discharge plasma actuator. Phys Fluids 25:017103

Jukes TN, Choi K-S (2013) On the formation of streamwise vortices by plasma vortex generators. J Fluid Mech 733:370-393

Kim J-H, Forte M, Choi K-S (2017) Report on the experimental results on transition delay in the "Juju" TRIN1 wind tunnel by the VR DBD actuators based on wall-normal jets. In: EU FP7 programme BUTERFLI deliverable D3.19, 3 May 2017

Kriegseis J, Simon B, Grundmann S (2016) Towards in-flight applications? A review on dielectric barrier discharge-based boundarylayer control. Appl Mech Rev 68:020802

Kurz HBE, Kloker MJ (2014) Receptivity of a swept-wing boundary layer to micron-sized discrete roughness elements. J Fluid Mech 755:62-82

Kurz HBE, Kloker MJ (2016) Mechanisms of flow tripping by discrete roughness elements in a swept-wing boundary layer. J Fluid Mech 796:158-194

Moralev I, Sherbakova V, Selivonin I, Bityurin V, Ustinov M (2018) Effect of the discharge constriction in DBD plasma actuator on the laminar boundary layer. Int J Heat Mass Transf 116:1326-1340

Moreau E (2007) Airflow control by non-thermal plasma actuators. J Phys D Appl Phys 40(3):605-636

Reed HL, Saric WS (1989) Stability of three-dimensional boundary layers. Annu Rev Fluid Mech 21:235-284

Reibert MS, Saric WS, Carrillo RB, Chapman JR K (1996) Experiments in nonlinear saturation of stationary crossflow vortices in a swept-wing boundary layer. In: AIAA paper 1996-0184

Santhanakrishnan A, Jacob JD (2007) Flow control with plasma synthetic jet actuators. J Phys D Appl Phys 40:637-651

Saric W, Carrillo RJR, Reibert M (1998) Leading-edge roughness as a transition control mechanism. In: AIAA paper 1998-781

Saric WS, Reed HL, White EB (2003) Stability and transition of threedimensional boundary layers. Annu Rev Fluid Mech 35:413-440 
Schuele CY (2010) Control of stationary crossflow modes using passive patterned roughness and DBD plasma actuators at Mach 3.5. $\mathrm{PhD}$ thesis, University of Notre Dame

Schuele CY, Corke TC, Matlis E (2013) Control of stationary crossflow modes in a Mach 3.5 boundary layer using patterned passive and active roughness. J Fluid Mech 718:5-38

Serpieri J, Kotsonis M (2018) Conditioning of unsteady cross-flow instability modes using dielectric barrier discharge plasma actuators. Exp Therm Fluid Sci 93:305-318

Serpieri J, Venkata SY, Kotsonis M (2017) Conditioning of cross-flow instability modes using dielectric barrier discharge plasma actuators. J Fluid Mech 833:164-205

Shahriari N, Kollert MR, Hanifi A (2018) Control of a swept-wing boundary layer using ring-type plasma actuators. J Fluid Mech 844:36-60

Ustinov MV, Moralev IA, Sboev DS, Baranov SA (2017) Experimental study of cross-flow dominated transition control by DRE-type

\section{Affiliations}

\section{Kwing-So Choi ${ }^{1}$ • Jung-Hoon Kim ${ }^{1}$}

\section{Kwing-So Choi}

kwing-so.choi@nottingham.ac.uk actuator. In: EU FP7 programme BUTERFLI deliverable D3.20, 27 April 2017

Venkata SY (2016) Control of stationary crossflow instability using DBD plasma actuators. MS thesis, TU Delft

Wang J-J, Choi K-S, Feng L-H, Jukes TN (2013) Recent developments in DBD plasma flow control. Prog Aerosp Sci 62:52-78

Wassermann P, Kloker M (2002) Mechanisms and passive control of crossflow-vortex-induced transition in a three-dimensional boundary layer. J Fluid Mech 456:49-84

Westerweel J (1997) Fundamentals of digital particle image velocimetry. Meas Sci Technol 8:1379-1392

Publisher's Note Springer Nature remains neutral with regard to jurisdictional claims in published maps and institutional affiliations.

Faculty of Engineering, University of Nottingham, Nottingham NG7 2RD, UK 